VOL. 61 (2000) [473-488]

\title{
ON THE NOTION OF COMPACTNESS IN SUPERGEOMETRY
}

\author{
Ugo Bruzzo and Vladimir Pestov
}

\begin{abstract}
We discuss the problem of finding an analogue of the concept of topological space in supergeometry, motivated by the search for a procedure to compactify supermanifolds along odd coordinates. In particular, we examine the topologies arising naturally on the sets of points of locally ringed superspaces, and show that in the presence of a nontrivial odd sector such topologies are never compact. The main outcome of our discussion is the following new observation: not only the usual framework of supergeometry (the theory of locally ringed spaces), but the more general approach of the functor of points, need to be further enlarged.
\end{abstract}

\section{INTRODUCTION}

Geometries with anticommuting variables (supergeometries) have been considered in connection with several issues in theoretical physics, notably to study supersymmetric field theories and superstring theory; some physical motivation for their introduction is discussed in [2]. Supergeometries were quite intensively studied in the 1970s and 1980s.

One natural question to ask is what are the proper analogues of the major concepts of topology for such geometries. In particular, how can we find 'topologies' which are capable of carrying information about the structure of superspaces 'in the odd directions'? This is not an idle question, as, for example, finding the right 'super' analogue of compactness and the ways to compactify supermanifolds are likely to have an impact on the formulation of some physical theories. (As a major example we cite the issue of the compactification of the moduli space of super Riemann surfaces in connection with superstring theory.) And the lack of a satisfactory cohomology theory for superspaces is just another manifestation of our failure to conceive (super)geometric objects which exhibit nontrivial topological structure in their odd sector.

We begin by outlining the sheaf-theoretic setting of supermanifold theory, which serves as the basis for supergeometry, whose key notion is that of a locally ringed superspace.

Received February 21, 2000

We thank C. Bartocci for valuable discussions. The research the present paper is based on was supported by SISSA, by the National Group for Mathematical Physics of CNR (both Italy), by the Internal Grants Committee of the Victoria University of Wellington, and by the Marsden Fund grant of the Royal Society of New Zealand 'Foundations of supergeometry.'

Copyright Clearance Centre, Inc. Serial-fee code: 0004-9727/00 \$A2.00+0.00. 
Next we address the problem, first explicitly stated by Leĭtes and Manin, of finding supergeometric analogues of compactness. In supergeometry, to every superspace there is associated a covariant functor from the category, $\mathbf{G}$, of all finite dimensional Grassmann algebras and graded-preserving algebra homomorphisms, to the category Sets of all sets and mappings. Such functors, $\mathfrak{X}$, are termed virtual superspaces. The image of a Grassmann algebra, $\Lambda(q)$, of rank $q$ under a virtual superspace functor, $\mathfrak{X}$, is denoted by $\mathrm{pt}_{q}(\mathfrak{X})$ and called the set of $q$-points of $\mathfrak{X}$. Let Top denote the category of all topological spaces and continuous mappings. It is quite natural to take as a basic concept of 'supertopology' that of a virtual topological superspace, that is, an object of the category Top $^{\mathbf{G}}$, formed by all covariant functors from $\mathbf{G}$ to Top and the corresponding functorial morphisms. We shall show that if $\mathfrak{X}$ is a locally ringed superspace and the ground field $k$ is topological (as is the case in all the standard examples), then for every $q \in \mathbb{N}$ the set of $q$-points of $\mathfrak{X}$ carries a natural topology. Therefore, a virtual superspace associated to an arbitrary locally ringed superspace has in fact a richer structure-that of a virtual topological superspace. We shall show, however, that a virtual topological superspace determined by a locally ringed superspace and having a nontrivial odd sector is never 'supercompact.' At best, such spaces are locally compact.

The category of virtual topological superspaces possesses natural compactifications; however, it seems that some of the most interesting conjectural objects of 'supertopology', such as the hypothetical 'purely odd projective space', do not correspond to objects of this category.

Our results suggest that, in order to embrace the phenomenon of compactness, the existing framework has to be further extended.

\section{GRADED ALGEBRA PRELIMINARIES}

Let $k$ denote an arbitrary field. We shall assume all algebras (over $k$ ) to be associative and unital. The word graded will be always synonymous with $\mathbb{Z}_{2}$-graded.

A graded vector space is a vector space $E$ together with a fixed direct sum decomposition $E=E_{0} \oplus E_{1}$. Elements of $E_{0}$ are said to be even, while elements of $E_{1}$ are referred to as odd. For any element $x \in E_{0} \cup E_{1}$ one denotes by $\widetilde{x} \in \mathbb{Z}_{2}$ the parity of $x$, determined by the rule $x \in E_{\tilde{x}}$ and computed mod 2. Elements of $E_{0} \cup E_{1}$ are called homogeneous. An example is supplied by the 'arithmetical' graded vector space $k^{m \mid n}$, which is just the vector space $k^{m+n}$ equipped with the grading $\left(k^{m \mid n}\right)_{0}=k^{m},\left(k^{m \mid n}\right)_{1}=k^{n}$.

Most of the basic constructions of linear algebra extend to the graded case and, in particular, direct sums and tensor products of graded vector spaces carry a natural (and often self-evident) grading. For details, we refer the reader to $[17,2]$.

A graded algebra is an algebra carrying a structure of a graded vector space, $A=$ $A_{0} \oplus A_{1}$. The two structures are required to agree with each other in the sense that for every $x, y \in A_{0} \cup A_{1}$ one has $\widetilde{x y}=\widetilde{x}+\widetilde{y}$. An associative unital graded algebra 
$A=A_{0} \oplus A_{1}$ is called graded commutative if for all $x, y \in A_{0} \cup A_{1}$ one has $x y=(-1)^{\widetilde{x} y} y x$. In particular, every commutative unital associative algebra, $A$, equipped with the trivial grading $\left(A_{0}=A, A_{1}=(0)\right)$, yields an example of a graded commutative algebra.

An ideal $I \subseteq A$ of a graded algebra $A$ is called graded if $I=I_{0} \oplus I_{1}$, where $I_{i}=I \cap A_{i}$, $i=1$,2. A graded commutative algebra $A$ is called local if it contains a unique maximal proper graded ideal $M$; the quotient $A / M$, called the residue field of $A$, is a field extension of the ground field $k$.

The most important example of a local graded commutative algebra is provided by the exterior, or Grassmann, algebra $\Lambda(q)$ of rank $q$-the associative unital algebra freely generated by $q$ pairwise anticommuting elements $\zeta_{1}, \zeta_{2}, \ldots, \zeta_{q}$; every element of $\Lambda(q)$ is a polynomial in the variables $\zeta_{i}$ with coefficients from $k$, having the form

$$
\sum_{\alpha \in 2^{(1, \ldots, q\}}} a_{\alpha} \zeta^{\alpha}
$$

where $\alpha \subseteq\{1,2, \ldots, q\}, \zeta^{\alpha}=\zeta_{\alpha_{1}} \zeta_{\alpha_{2}} \ldots \zeta_{\alpha_{|\alpha|}}$, and $\zeta^{\emptyset}=1$. Generators are subject to the anticommutation relations

$$
\zeta_{i} \zeta_{j}=-\zeta_{j} \zeta_{i} \text { for all } i, j=1, \ldots, q .
$$

Every collection of $q$ odd elements $\alpha_{1}, \ldots, \alpha_{q}$ of an arbitrary graded commutative (unital associative) algebra $A$ determines a unique graded algebra homomorphism from $\Lambda(q)$ to $A$ with $\zeta_{i} \mapsto \alpha_{i}$, called an $A$-point of $\Lambda(q)$.

An element of $\Lambda(q)$ is even (odd) if it can be represented as a sum of monomials in the free anticommuting generators $\zeta_{i}$ having even (odd) degree. The maximal graded ideal $N$ of $\Lambda(q)$ consists of all nilpotent elements, which are exactly those polynomials $p(\zeta)$ having vanishing constant term. The augmentation homomorphism (also called, in supergeometric jargon, the body map) is the quotient homomorphism $\beta: \Lambda(q) \rightarrow \Lambda(q) / N \cong k$, associating to every polynomial (2.1) the constant term $a_{\emptyset}$.

The following result shows that the Grassmann algebra of rank one $\Lambda(1)$ is a sense 'large enough' to be the target for a surjective homomorphism from every subalgebra of a Grassmann algebra with a nontrivial odd sector.

Lemma 2.1. Let $A$ be a graded unital subalgebra of a finite dimensional Grassmann algebra $\Lambda(q)$. If the odd part of $A$ is nontrivial, then there is a surjective graded algebra homomorphism $h: A \rightarrow \Lambda(1)$.

Proof: Define $m$ as the smallest cardinality of a subset $\beta \subseteq\{1,2, \ldots, q\}$ such that in the expansion (2.1) of at least one odd element $a \in A$ one has $a_{\beta} \neq 0$. It follows from our assumptions on $A$ that $m$ is positive and odd. Fix such a $\beta$.

Denote by $\chi$ a fixed odd generator of the Grassmann algebra $\Lambda(1)$ and define a 
grading-preserving $k$-linear mapping $\Lambda(q) \rightarrow \Lambda(1)$ by the rule

$$
\sum_{\alpha \in 2\{1, \ldots, q\}} \dot{a}_{\alpha} \zeta^{\alpha} \mapsto a_{\emptyset}+a_{\beta} \chi
$$

The image of $A$ under the above mapping is all of $\Lambda(1)$, and thus it suffices to prove that its restriction to $A$, say $h$, is an algebra homomorphism. (It is easy to see that in general the linear map (2.2) is not a homomorphism on all of $\Lambda(q)$.)

Let $x, y \in A$ be arbitrary. Represent $x=x_{0}+x_{1} \zeta^{\beta}+x_{2}, y=y_{0}+y_{1} \zeta^{\beta}+y_{2}$, where $x_{0}, y_{0} \in k$, and in the expansion (2.1) of $x_{2}$ and $y_{2}$ both the constant terms and the terms of order $\beta$ vanish. One has

$$
h(x)=x_{0}+x_{1} \chi, \quad h(y)=y_{0}+y_{1} \chi
$$

and consequently

$$
h(x) h(y)=x_{0} y_{0}+\left(x_{0} y_{1}+x_{1} y_{0}\right) \chi .
$$

At the same time,

$$
\begin{aligned}
x y= & \left(x_{0}+x_{1} \zeta^{\beta}+x_{2}\right)\left(y_{0}+y_{1} \zeta^{\beta}+y_{2}\right) \\
= & x_{0} y_{0}+\left(x_{0} y_{1}+x_{1} y_{0}\right) \zeta^{\beta} \\
& \quad+x_{0} y_{2}+y_{0} x_{2}+x_{1} y_{1} \cdot 0+\left(x_{1} y_{2}+x_{2} y_{1}\right) \zeta^{\beta}+x_{2} y_{2} .
\end{aligned}
$$

We claim that the image under $h$ of the terms in the last line of the above formula is zero, which finishes the proof. Firstly, since $x_{0}, y_{0} \in k$ and $h\left(x_{2}\right)=h\left(y_{2}\right)=0$, it follows from the linearity of $h$ that $h\left(x_{0} y_{2}+y_{0} x_{2}\right)=0$. Both $x_{2}$ and $y_{2}$ have no constant term, which implies that the term of order $\beta$ in the expansion (2.1) of $\left(x_{1} y_{2}+x_{2} y_{1}\right) \zeta^{\beta}$ vanishes (as well as the constant term of course) and, as a consequence, $h\left(\left(x_{1} y_{2}+x_{2} y_{1}\right) \zeta^{\beta}\right)=0$. Finally, $x_{2} y_{2}$ has no constant term and it cannot have a nonvanishing term of order $\beta$ either, because otherwise either $x_{2}$ or $y_{2}$ would contain a monomial of the form $a_{\gamma} \zeta^{\gamma}$ with $a_{\gamma} \neq 0,|\gamma|$ odd, and $|\gamma|<m=|\beta|$, which is impossible by the choice of $\beta$.

A very detailed treatment of Grassmann algebras from the viewpoint of supergeometry is to be found in the book [4].

\section{BASIC NOTIONS OF SUPERGEOMETRY}

3.1. Generalities The notions of presheaf and sheaf of graded algebras on a topological space, and that of stalk of a such a (pre)sheaf, are defined as usual, by requiring all the morphisms involved to be morphisms of graded algebras. For the basics of sheaf theory, the reader may consult, for example, [10].

A locally ringed superspace (else: geometric superspace) over $k$ is a pair $\mathfrak{X}=(X, \mathcal{S})$, where $X$ is a topological space and $\mathcal{S}_{X}$ is a sheaf of local graded commutative $k$-algebras. 
The latter means, through a slight abuse of language, that for every $V \neq \emptyset, \mathcal{S}(V)$ is a local graded commutative algebra, and that every stalk $\mathcal{S}_{X, x}$ is a local graded commutative algebra. The unique maximal ideal of $\mathcal{S}_{X, x}$ will be denoted by $m_{X, x}$, and the corresponding residue field $\mathcal{S}_{X, x} / m_{X, x}$ by $k_{X}(x)$ or $k(x)$. Sections $f \in \mathcal{S}(U)$ of the structure sheaf of a geometric superspace are called superfunctions on an open set $U$.

A morphism of geometric superspaces, $\phi: \mathfrak{X} \rightarrow \mathfrak{Y}, \phi=\left(\phi_{0}, \phi^{\sharp}\right)$, is formed by a continuous map $\phi_{0}: X \rightarrow Y$, and a local morphism of sheaves of $k$-algebras $\phi^{\natural}: \mathcal{S}_{Y} \rightarrow$ $\phi_{0, *}\left(\mathcal{S}_{X}\right)$; locality means that for every $x \in X$ the induced homomorphism between the stalks, $\phi_{x}^{\sharp}: \mathcal{S}_{Y, \phi_{0}(x)} \rightarrow \mathcal{S}_{X, x}$, satisfies the condition $\phi_{x}^{\sharp}\left(m_{Y, \phi_{0}(x)}\right) \subseteq m_{X, x}$. Here $\phi_{0, *}\left(\mathcal{S}_{X}\right)$ is the direct image sheaf on $Y$.

Let $\mathfrak{X}=(X, \mathcal{S})$ be a geometric superspace, and let $U$ be a nonempty open subset of $X$. Then $\left(U,\left.\mathcal{S}\right|_{U}\right)$ is a geometric superspace, which one may call an open geometric subsuperspace of $\mathfrak{X}$.

For a section $f \in \mathcal{S}_{X}(U)$ over an open set $U \subseteq X$ and for any $x \in U$ one can define the value of $f$ at $x$, denoted by $f(x)$, as the image under the augmentation homomorphism $\mathcal{S}_{X, x} \rightarrow k(x)=\mathcal{S}_{X, x} / m_{x}$. The necessity to introduce the functor of points (see the next section) stems from the fact that superfunctions-and therefore morphisms between superspaces-are not uniquely determined by the collection of their values.

Every locally ringed superspace, $\mathfrak{X}=(X, \mathcal{S})$, has a reflection in the category of locally ringed spaces and their morphisms, which we shall denote by $\mathfrak{X}_{\text {even }}$. The underlying topological space of $\mathfrak{X}_{\text {even }}$ is $X$, and for every open $U \subseteq X$ the algebra of sections is just $\mathcal{S}(U)_{0}$. The pair formed by the identity mapping of $X$ and the embedding of the sheaf $\mathcal{S}_{0}$ into $\mathcal{S}$ forms a superspace morphism from $\mathfrak{X}$ to $\mathfrak{X}_{\text {even }}$, which we shall denote by $r_{\text {even }}$. It has the following universal property: for every purely even locally ringed superspace $\mathfrak{Y}$ and every superspace morphism $f: \mathfrak{X} \rightarrow \mathfrak{Y}$ there exists a unique morphism of locally ringed spaces $\bar{f}: \mathfrak{X}_{\text {even }} \rightarrow \mathfrak{Y}$ such that $f=\bar{f}$ or $r_{\text {even }}$.

Every locally ringed superspace, $\mathfrak{X}$, has also a purely even coreflection, denoted by $\mathfrak{X}_{\text {red }}$ and called the reduced subsuperspace of $\mathfrak{X}$. There is a canonical morphism $i: \mathfrak{X}_{\text {red }} \rightarrow$ $\mathfrak{X}$ such that every morphism from a purely even geometric superspace, $\mathfrak{Y}$, to $\mathfrak{X}$, factors through $i$. The structure sheaf of $\mathfrak{X}_{\text {red }}$ is the quotient sheaf of $\mathcal{S}_{X}$ by the sheaf of ideals generated by the odd sector of $\mathcal{S}_{X}$.

3.2. SUPERMANIFOLDS An important example of a locally ringed superspace over $k=$ $\mathbb{R}$ is provided by a graded domain $U^{m, n}$ of dimension $(m, n)$, where $m, n$ are natural numbers. Its underlying topological space is an open domain, $U$, in an $m$-dimensional Euclidean space, while the structure sheaf is isomorphic to the sheaf of germs of infinitely smooth mappings from $U$ to the Grassmann algebra of rank $n$. In other words, for every open subset $V \subset U$ the graded algebra of superfunctions on $V, \mathcal{S}(V)$, is isomorphic to the graded tensor product $\mathcal{C}^{\infty}(V) \otimes \Lambda(n) \cong \mathcal{C}^{\infty}(V, \Lambda(n))$, where $\mathcal{C}^{\infty}$ is the sheaf of smooth real-valued functions on $U \subset \mathbb{R}^{m}$. 
By definition one has an epimorphism of sheaves of $\mathbb{R}$-algebras $\pi: \mathcal{S} \rightarrow \mathcal{C}^{\infty}$, and for every $x \in U$, the maximal ideal of $\mathcal{S}_{x}$ is the inverse image of the maximal ideal of $\mathcal{C}_{x}^{\infty}$ under $\pi$. The kernel of $\pi$ coincides with the sheaf of nilpotents of $\mathcal{S}$, which we denote $\mathcal{N}$.

If $n=0$, the definition of graded domain is identical with that of smooth domain of dimension $m$. At the other end there is the case $m=0$, leading to a 'purely odd' superspace which we shall denote by $\mathrm{pt}_{n}$. Its underlying topological space is a singleton, $\{*\}$, while the constant structure sheaf is $\Lambda(n)$. The $(0,0)$-dimensional superdomain $\mathrm{pt}_{0}$ is just a singleton considered as a trivial smooth manifold. One can introduce the concept of the spectrum of an arbitrary graded-commutative algebra $A$, very much in the same fashion as is done in algebraic geometry for commutative algebras; then the superspace $\mathrm{pt}_{q}$ is exactly $\operatorname{Spec} \Lambda(q)[14]$.

If $U=\mathbb{R}^{m}$ and $n$ is a fixed natural number, the corresponding graded domain is called a Euclidean superspace and is denoted $\mathbb{R}^{m, n}$.

A (real) smooth finite dimensional supermanifold (graded manifold), $\mathfrak{X}$, of dimension $(m, n)$ is a geometric superspace over the ground field $k=\mathbb{R}$ that is locally isomorphic to an $(m, n)$-dimensional graded domain. The underlying topological space of $\mathfrak{X}$ is a smooth manifold $X$ of dimension $m$. Every superdomain is a supermanifold. Every smooth manifold is a supermanifold of dimension $(m, 0)$. If $\mathfrak{X}=(X, \mathcal{S})$ is an $(m, n)$ dimensional supermanifold, one has an epimorphism of sheaves of $\mathbb{R}$-algebras $\pi: \mathcal{S} \rightarrow \mathcal{C}_{X}^{\infty}$, whose kernel is the nilpotent subsheaf $\mathcal{N}$ of $\mathcal{S}$. The quotient sheaf $\mathcal{N} / \mathcal{N}^{2}$ is a rank $n$ locally free $\mathcal{C}_{X}^{\infty}$-module, that is, it is the sheaf of sections of a rank $n$ vector bundle.

Morphisms between supermanifolds are just the geometric superspace morphisms described above. Thus, supermanifolds form a full subcategory of that of locally ringed superspaces and their morphisms.

3.3. Global StRUCTURE of SUPERmanifoldS If $E$ is a rank $n$ vector bundle on an $m$-dimensional differentiable manifold $X$, and $\mathcal{E}$ is the sheaf of sections of $E$, let $\mathcal{S}=\Lambda \mathcal{E}$ be the exterior algebra sheaf of $\mathcal{E}$, that is, the sheaf of sections of $\Lambda E$. It is quite easy to check that $(X, \mathcal{S})$ is an $(m, n)$ dimensional supermanifold. The vector bundle that, according to our previous discussion, can be associated to $(X, \mathcal{S})$, is straightforwardly proved to be isomorphic to $E$. We may wonder whether this construction is general, in the sense that, given a supermanifold $(X, \mathcal{S})$, the sheaf $\mathcal{S}$ is globally isomorphic to the exterior algebra sheaf of $\mathcal{N} / \mathcal{N}^{2}$. This is indeed true, and this is usually known as Batchelor's theorem (see, for example, [2]). The isomorphisms between $\mathcal{S}$ and $\Lambda\left(\mathcal{N} / \mathcal{N}^{2}\right)$ are in a one-to-one correspondence with the sections of the epimorphism $\pi: \mathcal{S} \rightarrow \mathcal{C}_{X}^{\infty}$, namely, with the morphisms of sheaves of $\mathbb{R}$-algebras $\sigma: \mathcal{C}_{X}^{\infty} \rightarrow \mathcal{S}$ such that $\pi \circ \sigma=\mathrm{id}_{\mathcal{C}_{X}^{\infty}}$. The validity of Batchelor's theorem relies on the fact that the structure sheaf $\mathcal{S}$ of a (real) supermanifold has trivial Čech cohomology since it admits partitions of unity.

Meticulous introductions to locally ringed superspaces and supermanifolds (graded manifolds) may be found in $[4,12,13,14,15,16,2]$. 


\section{FUNCTOR OF POINTS}

The functor of points traces its origins to algebraic geometry. Here we shall present it in the form it has assumed in supergeometry. Let $\mathfrak{X}$ be an arbitrary superspace, and let $q \in \mathbb{N}$. A $q$-point of $\mathfrak{X}$ is any superspace morphism $\xi: \mathrm{pt}_{q} \rightarrow \mathfrak{X}$. We shall first establish the following analogue of a well-known result holding for locally ringed spaces [8, p.119].

Proposition 4.1. Let $\mathfrak{X}=(X, \mathcal{S})$ be a locally ringed superspace over an arbitrary field $k$. The 0 -points of $\mathfrak{X}$ are in a natural one-to-one correspondence with the points $x \in X$ having $k$ as their residue field ('rational points').

PROOF: Since every unital algebra homomorphism between fields is an isomorphism, the image of $\{*\}$ under a 0 -point must have $k$ as its residue field. On the other hand, a morphism $\mathrm{pt}_{0} \rightarrow \mathfrak{X}$ is uniquely determined by the choice of the underlying mapping $\{*\} \rightarrow X$, which is in turn given by selecting a point in $X$.

The origin of this terminology is clarified by the remark that if $\mathfrak{X}=(X, \mathcal{S})$ is a supermanifold, then 0-points of $\mathfrak{X}$ are in a natural one-to-one correspondence with the points of the underlying smooth manifold of $X$. This follows from the isomorphism $\mathcal{S}_{x} \simeq \mathcal{C}_{x}^{\infty} \otimes \Lambda(n)$ holding for every $x \in X$ (here $\mathcal{C}^{\infty}$ is the sheaf of $C^{\infty}$ functions on $X$, and $n$ is the odd dimension of $\mathfrak{X}$ ).

Denote the collection of all $q$-points of $X$ by $\mathrm{pt}_{q}(X)$. The following is obvious from this definition.

Proposition 4.2. Let $U$ be an open subsuperspace of a locally ringed superspace $X$. Then for every $q \in \mathbb{N}$ the set $\mathrm{pt}_{q}(U)$ is a subset of $\mathrm{pt}_{q}(X)$ in a natural way.

Remark 4.3. A superspace need not have $q$-points at all. Using Proposition 4.1, it is easy to construct a nontrivial geometric superspace $X$ such that for every $q$, the set $\mathrm{pt}_{q}(X)$ is empty, see, for example, a similar example in [19].

A morphism of graded algebras $\varphi: \Lambda(q) \rightarrow \Lambda(p)$ determines a superspace morphism $\varphi^{\sharp}: \mathrm{pt}_{p} \rightarrow \mathrm{pt}_{q}$. If now $\xi: \mathrm{pt}_{q} \rightarrow \mathfrak{X}$ is a $q$-point of a locally ringed superspace $\mathfrak{X}$, then the composition $\xi \circ \varphi^{\sharp}$ is a $p$-point of $\mathfrak{X}$. Thus, $\varphi$ determines a mapping

$$
\varphi(\mathfrak{X}): \mathrm{pt}_{q}(\mathfrak{X}) \rightarrow \mathrm{pt}_{p}(\mathfrak{X})
$$

having the form

$$
\mathrm{pt}_{q}(\mathfrak{X}) \ni \xi \mapsto \xi \circ \varphi^{\sharp} \in \mathrm{pt}_{p}(\mathfrak{X}) .
$$

Using this observation, it is easy to verify that the correspondence

$$
\Lambda(q) \mapsto \mathrm{pt}_{q}(X), \quad \varphi \mapsto \varphi(\mathfrak{X})
$$


from the category $G$ to the category Sets is a covariant functor, which is of course representable by its very definition, with $X$ as the representing object:

$$
\mathrm{pt}_{q}(X)=\operatorname{Hom}\left(\mathrm{pt}_{q}, X\right)
$$

Definition 4.4: Denote by Sets ${ }^{\mathbf{G}}$ the category formed by all covariant functors $\mathfrak{X}: \mathbf{G} \rightarrow$ Sets and naturally defined functorial morphisms between them. Objects of this category, $\mathfrak{X}$, are called virtual superspaces. To keep the notation consistent, we shall denote the image of $\Lambda(q)$ under a functor $\mathfrak{X}$ by $\mathrm{pt}_{q}(\mathfrak{X})$. A morphism from a virtual superspace $\mathfrak{X}$ to a virtual superspace $\mathfrak{Y}$ (a functorial morphism) is a collection of mappings $f_{n}: \mathrm{pt}_{n}(\mathfrak{X}) \rightarrow \mathrm{pt}_{n}(\mathfrak{Y}), n \in \mathbb{N}$, commuting with mappings between the sets of points induced by each morphism between Grassmann algebras: a morphism $h: \Lambda(n) \rightarrow \Lambda(m)$ induces mappings $h(\mathfrak{X}): \mathrm{pt}_{n}(\mathfrak{X}) \rightarrow \mathrm{pt}_{m}(\mathfrak{X})$ and $h(\mathfrak{Y}): \mathrm{pt}_{n}(\mathfrak{Y}) \rightarrow \mathrm{pt}_{m}(\mathfrak{Y})$, and the requirement making $f$ into a functorial morphism is that $f_{m} \circ h(\mathfrak{X})=h(\mathfrak{Y}) \circ f_{n}$.

By assigning to every locally ringed superspace $\mathfrak{X}$ the virtual superspace $[\Lambda(q) \mapsto$ $\left.\mathrm{pt}_{q}(\mathfrak{X})\right]$, one obtains a functor from the category of locally ringed superspaces to the category of virtual superspaces. Indeed, every superspace morphism $f: \mathfrak{X} \rightarrow \mathfrak{Y}$ gives rise to a collection of mappings $f_{q}: \mathbf{p t}_{q}(\mathfrak{X}) \rightarrow \mathrm{pt}_{q}(\mathfrak{Y})$ in a consistent way. Here

$$
f_{q}(\xi)=f \circ \xi \in \mathrm{pt}_{\boldsymbol{q}}(\mathfrak{Y})
$$

for every $q$-point $\xi$ of $\mathfrak{X}$.

For more on the relationship between smooth supermanifolds and the associated virtual superspaces, see, for example, $[6,25]$.

EXAMPlE 4.5. The set $\mathrm{pt}_{q}\left(\mathbb{R}^{m, n}\right)$ of $q$-points of the $(m, n)$-dimensional Euclidean superspace $\mathbb{R}^{m, n}$ is the even sector of the graded vector space $\Lambda(q) \otimes \mathbb{R}^{m \mid n}$, where $\mathbb{R}^{m \mid n}$ stands for the graded vector space $\mathbb{R}^{m} \oplus \mathbb{R}^{n}$. To put it otherwise, $\mathrm{pt}_{q}\left(\mathbb{R}^{m, n}\right)$ is the set of elements of the vector space $\left[\mathbb{R}^{m} \otimes \Lambda(q)_{0}\right] \oplus\left[\mathbb{R}^{n} \otimes \Lambda(q)_{1}\right]$.

REMARK 4.6. The image, $\mathrm{pt}_{A}\left(\mathbb{R}^{m, n}\right)$, under the functor of points determined by $\mathbb{R}^{m, n}$ of a 'grassmannian' algebra $A$ forms a graded $A$-module, $A^{m, n}$, which was routinely accepted as the basic object of superanalysis and supergeometry by many theoretical and mathematical physicists. The resulting approach to supergeometry is known as the DeWitt-Rogers approach, see $[9,21,11]$. The approach we are following here is known as the Berezin-Leites-Kostant approach, see $[5,12,13,14,4,17]$. A functorial link between the two approaches was pointed out by Leĭtes [14] and (independently) A. Schwarz $[22,23]$, and remains to date largely unexplored. A brief discussion can be found in [18] and [3]. See also a paper by Schmitt [24], containing an excellent account of the functor of points in supergeometry. An early reference is the Stockholm preprint [6]. Some nontraditional aspects of the functor of points in infinite dimensional geometry are discussed in [20]. Notice that an infinite-dimensional 'grassmannian' algebra $A$ usually 
carries a natural locally convex algebra topology which must be taken into account in the definitions; the emerging subtleties may be disruptive for the expected pattern of results, see [7].

REMARK 4.7. Some virtual superspaces are represented by actual geometric superspaces, while others are not. Examples of such virtual superspaces are described in Remark 6.6.

Virtual superspaces can be considered as 'shadows' of actual objects of supergeometry, or sometimes as 'blueprints' for those objects still to be constructed. They are of little use in themselves. The authors of [1] have stressed that the progress in some areas of mathematical physics is hampered by the fact that though some objects (say, supermoduli spaces) admit a pretty clear interpretation via the functor of points, there are no known 'genuinely geometric' objects of supergeometry representing them-while such objects, and not their 'shadows', are exactly what one needs for work.

\section{ViRTUAL TOPOLOGICAL SUPERSPACES}

We begin with an auxiliary construction. Let $\mathfrak{X}=(X, \mathcal{S})$ be a locally ringed superspace over a field $k$, let $q \in \mathbb{N}$, and let $f$ be a superfunction on $X$. For an arbitrary $\xi \in \mathrm{pt}_{q}(X)$, the sheaf morphism $\xi^{\sharp}$ is in essence a graded algebra homomorphism from the stalk $S_{X, \xi_{0}(*)}$ to the Grassmann algebra $\Lambda(q)$. (Here $*$ is the only element of the topological space underlying $\mathrm{pt}_{q}$.) Denote by $\tilde{f}_{\xi}$ the germ of $f$ at the point $\xi_{0}(*)$.

Let $f_{q}(\xi)=\xi^{\sharp}\left(\tilde{f}_{\xi}\right)$. This is an element of the algebra of global sections of $\mathrm{pt}_{q}$, which is isomorphic to the Grassmann algebra $\Lambda(q)$. As $\xi$ runs over the set of all $q$-points of $\mathfrak{X}$, we thus obtain a mapping

$$
f_{q}: \mathrm{pt}_{q}(\mathfrak{X}) \rightarrow \Lambda(q) .
$$

Notice that for $q=0$, the element $f_{0}(\xi)$ coincides with the value of $f$ at the point $\xi_{0}(*)$, that is, the image of the germ of $f$ under the augmentation homomorphism $\mathcal{S}_{X, \xi_{0}(*)} \rightarrow k$.

Definition 5.1: Let $k$ be a topological field, and let $\mathfrak{X}=(X, \mathcal{S})$ be a locally ringed superspace over $k$. For every natural number $q$, we define the natural topology on the set $\mathrm{pt}_{q}(\mathfrak{X})$ as the coarsest topology with the following property: for every open subset $U \subseteq X$ and every superfunction $f \in \mathcal{S}(U)$, the mapping $f_{q}: \mathrm{pt}_{q}(U) \rightarrow \Lambda(q)$ is continuous with respect to the subspace topology on $\mathrm{pt}_{q}(U)$ and the standard product topology the Grassmann algebra supports as a finite dimensional vector space over $k$.

It is easy to show that the natural topology is well defined on sets of $q$-points for every locally ringed superspace over an arbitrary topological field.

EXAMPLE 5.2. If the structure sheaf on a 'purely even' superspace $\mathfrak{X}=(X, \mathcal{S})$ is a subsheaf of the sheaf of germs of continuous $k$-valued functions on $X$, then the natural 
topology on $\mathrm{pt}_{0}(\mathfrak{X})$ is contained in that induced from $X$. In particular, if $\mathfrak{X}$ is either a Tychonoff topological space with the sheaf of germs of continuous functions, or a smooth manifold with the sheaf of germs of smooth real-valued functions, then $X$ coincides with the set of all 0-points and the natural topology on $X$ is identical with the initial topology.

EXAMPLE 5.3. The natural topology on the set of $q$-points $\mathrm{pt}_{q}\left(\mathrm{pt}_{p}\right) \cong\left(\Lambda(q)_{1}\right)^{p}$ is easily shown to coincide with the product topology.

EXAMPLE 5.4. An arbitrary topological space $X$ is made into a (purely even) locally ringed superspace by equipping it with the sheaf of germs of continuous real-valued functions endowed with the trivial (purely even) grading. The stalks, $\mathcal{S}_{x}$, of the locally ringed space thus defined admit no nontrivial $\mathbb{R}$-valued derivations, and consequently the only homomorphism $\mathcal{S}_{x} \rightarrow \Lambda(q)$ is the augmentation $f \mapsto f(x)$. Consequently, for every $q \in \mathbb{N}$, the set of $q$-points of $X$ admits a canonical bijection with $X$ itself, and the topology on $\mathrm{pt}_{q}(X)$ is the completely regular replica of the topology of $X$. In particular, if $X$ is Tychonoff, then $\mathrm{pt}_{q}(X)$ is canonically homeomorphic to $X$ itself for each $q$.

LEMMA 5.5. For every graded algebra morphism $\varphi: \Lambda(p) \rightarrow \Lambda(q)$, the corresponding mapping $\varphi(\mathfrak{X}): \mathrm{pt}_{p}(\mathfrak{X}) \rightarrow \mathrm{pt}_{q}(\mathfrak{X})$ is continuous with respect to the natural topologies on both spaces.

Proof: Let an open subset $U \subseteq X$ and $f \in \mathcal{S}(U)$ be arbitrary. Since the functions of the form $f_{q}: \mathrm{pt}_{q}(U) \rightarrow \Lambda(q)$ determine the topology on $\mathrm{pt}_{q}(\mathfrak{X})$, it is enough to verify that the 'pull-back' of $f_{q}$ on $\mathrm{pt}_{p}(U)$ by means of the mapping $\varphi(\mathfrak{X}): \mathrm{pt}_{p}(\mathfrak{X}) \rightarrow \mathrm{pt}_{q}(\mathfrak{X})$ is continuous. In other words, it suffices to check the continuity of the mapping

$$
\left.f_{q}\right|_{\mathbf{p t}_{q}(U)} \circ \varphi(\mathfrak{X}): \mathbf{p t}_{p}(U) \rightarrow \Lambda(q) .
$$

To this end, it is enough to notice that $f_{q} \circ \varphi(\mathfrak{X})$ is the composition of the continuous mapping $\left.f_{p}\right|_{\mathbf{p t}_{p}(U)}$ with the graded algebra homomorphism $\varphi$ which is also continuous being a linear mapping between finite dimensional $k$-vector spaces.

The following is straightforward.

PROPOSITION 5.6. The correspondence

$$
\mathrm{pt}_{q} \mapsto \mathrm{pt}_{q}(X), \quad \varphi \mapsto \varphi(\mathfrak{X})
$$

is a covariant functor from the category $\mathbf{G}$ to the category Top.

Definition 5.7: A covariant functor from $\mathbf{G}$ to Top will be called a virtual topological superspace. The category whose objects are the virtual topological superspaces, and whose morphisms are the corresponding functor morphisms, will be denoted by Top $^{\mathbf{G}}$.

LEMMA 5.8. For every morphism $f: \mathfrak{X} \rightarrow \mathfrak{Y}$ between two locally ringed susperpaces and for every $q \in \mathbb{N}$ the mapping $f_{q}: \mathrm{pt}_{q}(\mathfrak{X}) \rightarrow \mathrm{pt}_{q}(\mathfrak{Y})$ defined by formula (4.1) is continuous with respect to the natural topologies. 
Proof: For each open subset $U \subseteq Y$ and every superfunction $g \in \mathcal{S}_{Y}(U)$, the pull-back $g_{q} \circ f_{q}$ coincides with $\left(f^{\sharp}(g)\right)_{q}$, where $f^{\sharp}(g)$ is an element of the algebra of sections $f_{0, *}\left(\mathcal{S}_{X}\right)(U)$, canonically isomorphic to $\mathcal{S}_{X}\left(f_{0}^{-1}(U)\right)$, and is therefore continuous on $\operatorname{pt}_{q}\left(f_{0}^{-1}(U)\right)$.

The following is an immediate corollary.

PROPOSITION 5.9. The assignment of a virtual topological superspace to every locally ringed superspace described in Proposition 5.6 is functorial (in a covariant way).

The following structural result is very simple yet useful.

Propos ITION 5.10. Let $\mathfrak{X}$ be a virtual topological superspace. Then for every natural number $q$, the space $\mathrm{pt}_{q}(\mathfrak{X})$ is fibred over $\mathrm{pt}_{0}(\mathfrak{X})$ in a canonical manner. If $\mathfrak{X}$ is [determined by] a locally ringed superspace, then the fibre over $x$ is homeomorphic to $\operatorname{Hom}\left(\mathcal{S}_{x}, \Lambda(q)\right)$.

Proof: The augmentation homomorphism $\beta: \Lambda(q) \rightarrow k \cong \Lambda(0)$ determines a superspace morphism $\beta_{\bullet}: \mathrm{pt}_{0}(\mathfrak{X}) \rightarrow \mathrm{pt}_{q}(\mathfrak{X})$. The image of $\beta_{\text {}}$ under the functor $\mathfrak{X}$ is a continuous mapping and therefore supplies the desired canonical fibration $\beta_{\bullet}(\mathfrak{X}): \mathrm{pt}_{q}(\mathfrak{X}) \rightarrow$ $\mathrm{pt}_{0}(\mathfrak{X})$. The inclusion $\Lambda(0) \cong k \hookrightarrow \Lambda(q), \lambda \mapsto \lambda \cdot 1$ is a homomorphism of unital graded algebras and therefore determines a superspace morphism $i: \mathrm{pt}_{q} \rightarrow \mathrm{pt}_{0}$; one thus obtains a continuous mapping $i(\mathfrak{X}): \mathrm{pt}_{0}(\mathfrak{X}) \rightarrow \mathrm{pt}_{q}(\mathfrak{X})$. The obvious property $\beta \circ i=i$ implies that $\beta(\mathfrak{X}) \circ i(\mathfrak{X})=i(\mathfrak{X})$, that is, $\beta(\mathfrak{X})$ is a retraction of $\mathrm{pt}_{q}(\mathfrak{X})$ onto a subspace homeomorphic to $\mathrm{pt}_{0}(\mathfrak{X})$, and in particular all fibres are nonempty.

Now assume that $\mathfrak{X}$ is determined by a locally ringed superspace, which we shall for simplicity denote with the same symbol $\mathfrak{X}=(X, \mathcal{S})$. According to Proposition 4.1, 0 -points of $\mathfrak{X}$ correspond to the points $x \in X$ having $k$ as their residue field. It follows that if $\xi: \mathrm{pt}_{q} \rightarrow \mathfrak{X}$ is a $q$-point, then $\xi_{0}(*)=x \in X$ is a 0 -point of $X$, while $\xi^{\sharp}$ can be thought of as an arbitrary graded algebra homomorphism from the stalk $\mathcal{S}_{x}$ to $\Lambda(q)$. Notice that $\xi_{0}(*)$ is exactly $\beta(\mathfrak{X})(\xi)$. Therefore, the collection of all $q$-points $\xi$ with $\xi_{0}(*)=x$ coincides with the fibre of the canonical fibration $\beta_{\bullet}(\mathfrak{X}): \operatorname{pt}_{q}(\mathfrak{X}) \rightarrow \mathrm{pt}_{0}(\mathfrak{X})$ over the point $x$. Another way to describe this fibre is as the collection $\operatorname{Hom}\left(\mathcal{S}_{x}, \Lambda(q)\right)$ of all graded algebra homomorphisms from the stalk $\mathcal{S}_{x}$ to $\Lambda(q)$. The proof is thus finished.

\section{NONCOMPACTNESS OF LOCALLY RINGED TOPOLOGICAL SUPERSPACES}

It is natural to call a virtual topological superspace, $\mathfrak{X}$, compact if for every $q$, the topological space of $q$-points, $\mathrm{pt}_{q}(\mathfrak{X})$, is compact. However, here we shall show that the only occurrences of such a phenomenon are in a sense trivial, and thus, informally speaking, compactness along the odd directions is never observed among virtual topological superspaces. 
First of all, we need to define what it means that a virtual topological superspace determined by a locally ringed superspace is nontrivial in the odd sector.

DEFINITION 6.1: Let $\mathfrak{X}$ be a locally ringed superspace. We say that the virtual topological superspace determined by $\mathfrak{X}$ is trivial in the odd sector if for every $q \in \mathbb{N}$ and $\xi \in \mathrm{pt}_{q}(\mathfrak{X})$ the graded subalgebra $\xi^{\sharp}\left(\mathcal{S}_{x}\right)$ of $\Lambda(q)$ has trivial odd sector.

Here is an equivalent reformulation of the same concept. Recall that $i: \mathfrak{X}_{\text {red }} \rightarrow \mathfrak{X}$ is the canonical morphism from the reduced subsuperspace (even co-reflection) of $\mathfrak{X}$.

PRoposition 6.2. Let $\mathfrak{X}$ be a locally ringed superspace. The corresponding virtual topological superspace is trivial in the odd sector if and only if for every $q \in$ $\mathbb{N}$, the continuous mapping $i_{q}: \mathrm{pt}_{q}\left(\mathfrak{X}_{\mathrm{red}}\right) \rightarrow \mathrm{pt}_{q}(\mathfrak{X})$ is a homeomorphism. Equivalently, the functor associating a virtual topological superspace to $\mathfrak{X}$ factors through the even coreflection $\mathfrak{X}_{\text {red }}$.

Loosely put, this is the case where the odd sector of a superspace, $\mathfrak{X}$, tells us nothing about the topology on $q$-points that is not already encoded in the even subsuperspace $\mathfrak{X}_{\text {red }}$.

LEMMA 6.3. Let $\mathfrak{X}$ be a locally ringed superspace, and let $x \in \mathrm{pt}_{0}(\mathfrak{X})$ and $q \in \mathbb{N}$. The restriction of the natural topology to the fibre, $\operatorname{Hom}\left(\mathcal{S}_{x}, \Lambda(q)\right)$, of the canonical fibration $\mathrm{pt}_{q}(\mathfrak{X}) \rightarrow \mathrm{pt}_{0}(\mathfrak{X})$ over $x$ coincides with the topology induced from the Tychonoff topology on the infinite product $\Lambda(q)^{\mathcal{S}_{x}}$ under the embedding $\operatorname{Hom}\left(\mathcal{S}_{x}, \Lambda(q)\right) \hookrightarrow \Lambda(q)^{\mathcal{S}_{x}}$.

PRoOF: The natural topology on the fibre $\operatorname{Hom}\left(\mathcal{S}_{x}, \Lambda(q)\right)$, formed by all $q$-points $\xi$ with $\xi_{0}(*)=x$, is, by the definition, the coarsest topology making every mapping of the form $\xi \mapsto f(\xi)$ continuous, where $f \in \mathcal{S}(U)$, and $U$ is an arbitrary open neighbourhood of $x$. If $h_{\xi}$ is a homomorphism from the stalk $\mathcal{S}_{x}$ to $\Lambda(q)$ associated to $\xi$, then the natural topology is the coarsest one making every mapping of the form $\xi \mapsto h_{\xi}(\tilde{f})$ continuous, where $\widetilde{f}$ is the germ of a superfunction $f$ as above. This is exactly the topology of simple convergence on elements of the stalk $\mathcal{S}_{x}$, that is, the topology induced on $\operatorname{Hom}\left(\mathcal{S}_{x}, \Lambda(q)\right)$ from $\Lambda(q)^{\mathcal{S}_{x}}$, as required.

The following result shows that among virtual topological superspaces determined by locally ringed superspaces, every compact superspace is trivial in the odd sector, that is, it comes from a locally ringed space rather than superspace.

THEOREM 6.4. Let $k$ be an infinite topological field, and let $\mathfrak{X}=\left(X, \mathcal{S}_{X}\right)$ be a locally ringed topological superspace over $k$. Assume that the topological space $\mathrm{pt}_{1}(\mathfrak{X})$ (with the natural topology) is compact. Then the virtual topological superspace determined by $\mathfrak{X}$ is trivial in the odd sector.

PRoOF: Assume that the virtual topological superspace determined by $\mathfrak{X}$ is nontrivial in the odd sector, that is, there is a $q \in \mathbb{N}_{+}$and a $q$-point of $\mathfrak{X}, \xi$, such that $A=\xi^{\sharp}\left(\mathcal{S}_{x}\right)$ has nantrivial odd sector as a graded subalgebra of $\Lambda(q)$. According to 
Lemma 2.1, there is a surjective morphism of graded algebras $j: A \rightarrow \Lambda(1)$. Denote by $\beta: A \rightarrow k$ the restriction of the augmentation homomorphism $\Lambda(q) \rightarrow k$ to $A$. It is clear that for every even element $a_{0} \in A_{0}$ one must have $j\left(a_{0}\right)=\beta\left(a_{0}\right)$. From here it follows that for every value of the parameter $\lambda \in k$ the linear mapping $j_{\lambda}: A \rightarrow \Lambda(1)$ determined by $j_{\lambda}\left(a_{0}+a_{1}\right)=\beta\left(a_{0}\right)+\lambda j\left(a_{1}\right), a_{i} \in A_{i}, i=0,1$, is a graded algebra homomorphism. For two different values $\lambda_{1} \neq \lambda_{2}$, the homomorphisms $j_{\lambda_{1}}$ and $j_{\lambda_{2}}$ are distinct. Every composition of the form $j_{\lambda} \circ \xi^{\sharp}$ is a graded algebra homomorphism from $\mathcal{S}_{x}$ to $\Lambda(1)$, and therefore determines a 1-point of $\mathfrak{X}$, and, moreover, an element of the fibre of the fibration $\mathrm{pt}_{1}(\mathfrak{X}) \rightarrow \mathrm{pt}_{0}(\mathfrak{X})$ at the point $x$ (Proposition 5.10). We shall denote such a 1-point by $x_{\lambda}$. For different values of $\lambda$, the points $x_{\lambda}$ are different. It follows from Lemma 6.3 that the set of all points $\left\{x_{\lambda}: \lambda \in k\right\}$ equipped with the natural topology is canonically homeomorphic to the basic field $k$. Indeed, in the topology of pointwise convergence, a net $x_{\lambda_{\nu}}$ converges to a point $x_{\mu}$ if and only if for every $z \in \Lambda(q)$ the net $j_{\lambda_{\nu}}\left(\xi^{\sharp}(z)\right)$ converges to $j_{\mu}\left(\xi^{\sharp}(z)\right)$, which is easily shown to be equivalent to the convergence $\lambda_{\nu} \rightarrow \mu$. Moreover, the set $\left\{x_{\lambda}: \lambda \in k\right\}$ is readily verified to form a one-dimensional affine subspace in $\Lambda(q)^{S_{x}}$ and therefore is closed with respect to the (locally convex Hausdorff) Tychonoff product topology on $\Lambda(q)^{S_{x}}$. Since an infinite topological field is never compact [26], it means that the fibre of $\mathrm{pt}_{1}(\mathfrak{X})$ over $x$ is noncompact. But it is closed in $\mathrm{pt}_{1}(\mathfrak{X})$, which is a contradiction.

REMARK 6.5. Observe that the category of virtual topological superspaces possesses 'compactifications.' Suppose a virtual superspace, $\mathfrak{X}$, is 'Tychonoff' in the sense that for each $q$, the space $\mathrm{pt}_{q}(\mathfrak{X})$ is Tychonoff. Define for each $n \in \mathbb{N}$

$$
\operatorname{pt}_{n}(\beta \mathfrak{X}) \stackrel{\text { def }}{=} \beta\left(\mathrm{pt}_{n}(\mathfrak{X})\right),
$$

where $\beta$ denotes, as usual, the Stone-Cech compactification. Every continuous mapping $f: \mathrm{pt}_{n}(\mathfrak{X}) \rightarrow \mathrm{pt}_{m}(\mathfrak{X})$ extends to a unique continuous mapping $\tilde{f}: \beta\left(\mathrm{pt}_{n}(\mathfrak{X})\right) \rightarrow$ $\beta\left(\mathrm{pt}_{m}(\mathfrak{X})\right)$, which enables one to turn the correspondence $\Lambda(n) \rightarrow \beta\left(\mathrm{pt}_{n}(\mathfrak{X})\right)$ into a covariant functor and indeed a virtual topological superspace, containing $\mathfrak{X}$ as a virtual topological subsuperspace in a natural fashion.

Since every supermanifold $\mathfrak{X}$ is 'super-Tychonoff' in the sense that the natural topology on each set $\mathrm{pt}_{n} \mathfrak{X}$ is Tychonoff, it admits a nice compactification in the category of virtual topological superspaces.

The compactification procedure for virtual topological superspaces certainly deserves further attention. In particular, it enables one to produce numerous examples of virtual topological superspaces that do not come from locally ringed superspaces. Such is, for instance, the above described 'compactification' of [the virtual topological superspace assigned tol any supermanifold, $\mathfrak{X}$, whose odd dimension $n \neq 0$.

REMARK 6.6. It is, however, important to realise that the above compactification procedure does not provide an answer, or at least a complete answer, to the problem of 
compactifying supermanifolds - simply because some of the most intriguing hypothetical objects of supertopology, such as the purely odd projective superspace, do not correspond to any virtual topological superspace. The setting of functor of points is, thus, too restrictive.

This is the first time such an observation has been made. The often held viewpoint has previously been that the functor of points provided an adequate setting for the notion of compact superspace, and only translating this notion into a geometric language posed a problem. To quote from Leites's problem survey article [15, pp. 650-651]:

Consider for instance $G L(n)$ acting on the space of the identity representation. There are two orbits: the origin and the rest. If we now look at the space as an $(0, n)$ dimensional supermanifold we see that the complement to the origin is just a kind of halo, indescribable except in the language of the point functor. The functor corresponding to the complement of the origin is not presented by a supermanifold.

Functors on the category of commutative (super)algebras represented by manifolds or supermanifolds are good because you can construct differential or "at least" algebraic geometries on them. How to distinguish subfunctors corresponding to the orbits of supergroup action? (A similar problem takes place for groups and their orbits in prime characteristics.)

Is it possible to construct mechanics on such orbits, that is, integral and differential calculus?

In fact, the situation is even less favourable than appears from the above: the 'functor corresponding to the complement of the origin' referred to by Leites simply does not exist. If $\mathfrak{X}$ were such a virtual superspace, then one would have

$$
\mathrm{pt}_{0}(\mathfrak{X})=\mathrm{pt}_{0}\left(\mathrm{pt}_{q}\right) \backslash\{0\}=\emptyset
$$

and, by the covariance of $\mathfrak{X}$,

$$
\mathrm{pt}_{q}(\mathfrak{X}) \subseteq \beta(\mathfrak{X})^{-1}\left(\mathrm{pt}_{0}(\mathfrak{X})\right)=\emptyset
$$

for all $q$, where $\beta: \wedge(q) \rightarrow \wedge(0) \cong k$ is the homomorphism of augmentation.

We conclude that none of the known frameworks for supergeometry, not even the functor of points, allows for the existence of some of the most interesting objects one would like to see implemented in supertopology.

\section{REFERENCES}

[1] M.A. Baranov, Yu.I. Manin, I.V. Frolov and A.S. Schwarz, 'A superanalog of the Selberg trace formula and multiloop contributions for fermionic strings', Comm. Math. Phys. 111 (1987), 373-392.

[2] C. Bartocci, U. Bruzzo, and D. Hernández Ruipérez, The Geometry of Supermanifolds (Kluwer Academic Publ., Dordrecht, 1991). 
[3] C. Bartocci, U. Bruzzo, D. Hernández Ruipérez and V.G. Pestov, 'Supermanifold theory: towards a unifying approach', in Proc. 4th Fall Workshop on Differential Geometry and its Applications, (M. Salgado and E. Vázquez, Editors), Anales de Física, Monografías 3 (CIEMAT, Madrid, 1996), pp. 1-16.

[4] F.A. Berezin, Introduction to superanalysis, (A.A. Kirillov, Editor) (D. Reidel Publ. Co, Dordrecht, Boston MA, 1987).

[5] F.A. Berezin and D.A. Leĭtes, 'Supermanifolds', Soviet Math. Dokl. 16 (1975), 1218-1222.

[6] J. Bernstein and D.A. Leĭtes, 'Lie superalgebras and supergroups', Report Dept. Math. Univ. Stockholm 14 (1988), 132-260.

[7] U. Bruzzo and V. Pestov, 'On the structure of DeWitt supermanifolds', J. Geom. Phys. 30 (1999), 169-186.

[8] M. Demazure and P. Gabriel, Introduction to algebraic geometry and algebraic groups (North-Holland, Amsterdam, New York, Oxford, 1980).

[9] B.S. DeWitt, Supermanifolds (Cambridge University Press, London, 1984).

[10] R. Godement, Théorie des faisceaux (Hermann, Paris, 1964).

[11] A. Jadczyk and K. Pilch, 'Superspaces and supersymmetries', Comm. Math. Phys. 78 (1981), 373-390.

[12] B. Kostant, 'Graded manifolds, graded Lie theory, and prequantization', in Differential Geometric Methods in Mathematical Physics, Lecture Notes Math. 570 (Springer-Verlag, Berlin, Heidelberg, New York, 1977), pp. 177-306.

[13] D.A. Leites, 'Introduction to the theory of supermanifolds', Russian Math. Surveys 35 (1980), 1-74.

[14] D.A. Leĭtes, The theory of supermanifolds, (in Russian) (Karelian Branch Acad. Sciences U.S.S.R., Petrozavodsk, 1983).

[15] D.A. Leĭtes, 'Selected problems of supermanifold theory', Duke Math. J. 54 (1987), 649-656.

[16] Yu.I. Manin, 'New directions in geometry', Russian Math. Surveys 39 (1984), 47-73.

[17] Yu.I. Manin, Gauge field theory and complex geometry, Grundlehren der Mathematischen Wissenschaften 289 (Springer-Verlag, Berlin, Heidelberg, New York, 1988).

[18] I.B. Penkov, 'Classical Lie supergroups and their superalgebras and their representations', Ann. Inst. Fourier 117 (1988).

[19] V.G. Pestov, 'A contribution to nonstandard superanalysis', J. Math. Phys. 33 (1992), 3263-3273.

[20] V.G. Pestov, 'On even collective effects in purely odd superspaces', in Proc. XXIInd Int. Conf. on Differential Geometric Methods in Theoretical Physics, Adv. Appl. Clifford Alg. (Proc. Suppl.) 4, 1994, pp. 157-164.

[21] A. Rogers, 'A global theory of supermanifolds', J. Math. Phys. 21 (1980), 1352-1365.

[22] A.S. Schwarz, 'Supergravity, complex geometry and $G$-structures', Commun. Math. Phys. 87 (1982/83), 37-63.

[23] A.S. Schwarz, 'On the definition of superspace', Theor. Math. Phys. 60 (1984), 657-660.

[24] T. Schmitt, 'Supergeometry and quantum field theory, or: What is a classical configuration', Rev. Math. Phys. 9 (1997), 993-1052.

[25] A.A. Voronov, 'Mappings of supermanifolds', Theor. Math. Phys. 60 (1984), 660-664. 
[26] W. Więsław, Topological fields, Monographs and Textbooks in Pure and Applied Mathematics 119 (Marcel Dekker, New York, 1988).

Scuola Internazionale Superiore di

Studi Avanzati (SISSA)

Via Beirut 4

34014 Trieste

Italy

and

Dipartimento di Matematica, Università degli Studi di Genova

Via Dodecaneso 35

16146 Genova

Italy

e-mail: bruzzo@sissa.it
School of Mathematical and Computing Sciences Victoria University of Wellington

P.O. Box 600

Wellington

New Zealand

e-mail: vova@mcs.vuw.ac.nz 